\title{
Enhanced Recovery Following Bariatric Surgery: Is Feasible?
}

\author{
Fabiana Lucci ${ }^{1}$, Paolo Gentileschi ${ }^{2}$, Maria Beatrice Silvi ${ }^{1}$, Federico Perrone ${ }^{2}$, Mario Dauri ${ }^{1}$
}

'Department of Anesthesia and Intensive care, Tor Vergata University, Rome, Italy

${ }^{2}$ Department of General and Metabolic surgery, Tor Vergata University, Rome, Italy

\section{ABSTRACT}

Background: There has been a relative lack of research on the effect of applying enhanced recovery principles in the context of morbid obesity surgery and monitoring outcome.

Objectives: To determine the feasibility of applying enhanced recovery after surgery (ERAS) principles application on a bariatric population and review the effect on outcome in this population.

Methods: We analyzed data prospectively collected on a cohort of 111 patients undergoing laparoscopic bariatric surgery between March 2013 and December 2014. All patients were enrolled in an ERAS protocol and were assessed for fitness for early discharge. We focused our attention on earlycomplications (within 30 days) and any late complications.

Results: All 111 patients who underwent bariatric surgery were suitable for early discharge between 24-72 hours. Short term complications (within 30 days postoperatively) were acceptable. Mortality rate was $0 \%$, reoperation rate was $0 \%$ and hospital readmission rate was $2 \%$. Concerning the short term complications in the 63 patients who underwent LAGB the commonest was the raised temperature. Hospital readmission rate, reoperation rate and mortality were all $0 \%$.

Conclusion: Application of ERAS protocol on bariatric surgery is feasible with an acceptable number of minor complications. Further studies are required to improve outcome further.

Key words: bariatric surgery, laparoscopic sleeve gastrectomy (Isg), laparoscopic adjustable gastric banding (lagb), enhanced recovery after surgery (ERAS)

\section{INTRODUCTION}

Enhanced recovery after surgery (ERAS) protocols are multimodal perioperative care pathways designed to achieve early recovery after surgical procedures by maintaining preoperative organ function and reducing the profound stress response following surgery.

The key elements of ERAS protocols include pre-operative counseling, optimization of nutrition, standardised analgesic and anaesthetic regimens and early mobilization. (1-3)

Despite the significant body of evidence showing that ERAS protocols lead to improved outcomes, they challenge traditional surgical doctrine, and as a result their implementation has been slow (4).

Initiated by Professor Henrik Kehlet in the 1990s, ERAS, enhanced recovery
Corresponding author:

Fabiana Lucci, MD

Department of Anesthesia and

Intensive care, Tor Vergata University

Rome, Italy

E-mail: fabiana.lucci@libero.it
Received: 23.11.2016

Accepted: 10.12 .2016 
programs or fast-track programs have become an important focus of peri-operative management after colorectal surgery, vascular surgery, thoracic surgery and more recently bariatric surgery $(5,6,7,8,9)$.

Enhanced Recovery After Surgery (ERAS) pathways integrate multimodal perioperative interventions which are designed to reduce physiological stress, facilitate early return of body function and reduce healthcare costs by reducing length of hospital stay (LOS) (1). The use of enhanced recovery after surgery (ERAS) programs has been reported to reduce morbidity in various surgical specialties, particularly in the context of lower gastrointestinal surgery (10). On the other hand, there has been a relative dearth of research on this subject in bariatric surgery. A recent randomized clinical trial of ERAS versus standard care after LSG has reported a shortened in-hospital length of stay (LOS) and found ERAS to be cost effective with no increase in perioperative morbidity (11)

There is, however, paucity of ERAS data originating from patients undergoing bariatric and metabolic surgery (1). A recently reported randomized clinical trial examined an enhanced recovery pathway versus standard care following 78 patients undergoing laparoscopic sleeve gastrectomy (5). In the latter study patients in the ERAS group demonstrated significantly shorter LOS and reduced hospital costs with no increase in occurrence of complications, although there was a $20 \%$ readmission rate in both ERAS and control groups (5).

Reasons for the delayed adoption of ERAS pathways within bariatric centres may include lack of robust evidence within this group of patients and concerns regarding the presence of complex high-risk medical comorbidities that require specialist peri-operative care. The aims of the present study were, therefore, to determine the feasibility and clinical outcomes following implementation of an Enhanced Recovery After Bariatric Surgery (ERABS) pathway in a University Hospital.

\section{MATERIAL AND METHODS}

We analyzed data prospectively collected on 111 consecutive patients undergoing primary bariatric procedures, (sleeve gastrectomy LSG and gastric banding LAGB), within an ERABS pathway. Procedures were performed over a 21-months period (January 2013 to December 2014) at the University Hospital of Tor Vergata in Rome. The study had approval from the hospital ethics committee. All procedures performed were in accordance with the ethical standards of the institutional and/or national research committee and with the 1964 Helsinki declaration and its later amendments or comparable ethical standards.

When considering the suitability for discharge on the second postoperative day, each patient was required to understand the planned procedure and postoperative care and to definitely express willingness to engage in the early discharge process. Social and geographic factors were also taken into account: (a) A responsible adult needs to escort the patient home and provide support for the following 24 to 48 hours; (b) patient's domestic circumstances need to be appropriate for postoperative care (e.g., adequate familial support, presence of facilities on the ground floor, etc.); (c) the patient should live at a distance of a maximum 2-hour drive from the hospital.

Preoperative workup included routine blood tests, chest radiograph and an electrocardiogram, Barium $x$-ray of the upper gastrointestinal tract and/or esophagogastro-duodenoscopy (OGD). Echocardiogram, sleep studies, and abdominal ultrasound were performed on an individual basis depending on clinical history and physical findings. Psychiatric counseling was conducted to evaluate mental health contraindications to surgery and obstacles to postoperative success in order to identify patients unsuitable for surgery and early discharge.

\section{Inclusion criteria}

Inclusion criteria were: failure to lose weight via other methods, $\mathrm{BMI}>40.0 \mathrm{~kg} / \mathrm{m}^{2}$ or $\geq 35.0 \mathrm{~kg} / \mathrm{m}^{2}$ with obesity-related comorbidities, no previous bariatric surgery, no alcohol or drug issues, no active psychosis, no mental retardation with an IQ below 50 and no severe active uncontrolled psychiatric symptomatology, no presence of cardiac, renal or liver failure, no wheelchair-dependancy. Surgical procedures (LSG and LAGB) were selected on the base of the pre-operative work-up. All procedures were performed laparoscopically using four or five ports by the same surgeon. At our institution, LSG is performed with a $36-\mathrm{F}$ bougie and gastric resection is carried out with a reinforced linear stapler. LAGB is performed using a standard "pars Flaccida' approach. Nasogastric tubes and urinary catheters were not routinely used and use of surgical drains was reserved for patients whom the surgeon considered at increased postoperative risk of bleeding.

Low-molecular-weight heparin thromboprophylaxis and $\mathrm{H} 2$-blocker therapy were started 12 hours before the operation and were continued postoperatively for 2 and 12 weeks, respectively.

Since January 2013, perioperative care has been standardized in accordance with a locally agreed ERAS 
protocol. All patients and their family underwent a structured preoperative information meeting where they were educated about the physical and behavioral changes induced by the operation and were also taught how to manage postoperative expectations. Explicit preoperative information can facilitate postoperative recovery and pain control, particularly in patients exhibiting denial and anxiety. $(12,13,14)$ A clear explanation of expectations during hospitalization facilitates adherence to the care pathway and allows early recovery and discharge. At this first encounter, the patient should also be given a clear role with specific tasks, including targets for postoperative food intake, oral nutritional supplements, and mobilization. Anesthetic assessment involves careful evaluation of patient comorbidities and optimization of functional status.

\section{Anaesthetic protocol}

At induction of anesthesia all patients were preoxygenated and were given intravenous dexamethasone $8 \mathrm{mg}$ and antibiotic prophylaxis. Bispectral index monitoring was started before propofol-induced anesthesia. BIS is a scale derived from the measurement of cerebral electrical activity in anesthetized patients so that the level of anaesthesia and drug delivery can be optimized, with a faster postoperative recovery. Total intravenous anesthesia using a combination of remifentanil and propofol was administered, with rocuronium given to achieve complete muscle relaxation. Pressurecontrolled ventilation with a positive end-expiratory pressure (PEEP) at $5 \mathrm{~cm} / \mathrm{H}_{2} \mathrm{O}$ was used to prevent formation of atelectasis and improve oxygenation. Analgesia was started intra-operatively and includes the use of paracetamol, ketorolac and ropivacaine $0,25 \%$ intraperitoneal instillation, through OptiSpray TM. Effective Pain management is particularly relevant in obese patients who experience a higher number of cardiovascular and pulmonary events, so is really important reduce opioids requirement, in order to avoid respiratory depression. For the first 12 post-operative hours, all patients were prescribed a morphine sulfate patient-controlled analgesic (PCA) pump and maintenance intravenous crystalloids, administered at a rate of $0.5-1 \mathrm{~mL} / \mathrm{kg} / \mathrm{hr}$. Immediately after recovery from general anesthesia, they were allowed 30-mL sips of clear fluid every 15-20 minutes. Mobilization out of bed was encouraged on the same day of the operation, and scrupulous attention was paid to the hemodynamic parameters in the immediate postoperative period. Once the PCA was stopped, pain control was provided with oral multimodal analge- sia (i.e., paracetamol, nonsteroidal antiinflammatory drugs, and opioids). After discussion with the patient and the family, discharge is programmed.

Post-discharge advices included a diet consisting of clear liquids and puréed foods for 15 days after surgery and a semisolid-consistency diet for the next 15 days. After the first 30 days, patients gradually began a low-fat, low-carbohydrate, high-protein solid diet based on the advice of a dietitian. One multivitamin tablet, daily intake, was recommended for three months after surgery, as well as high-frequency water intake and physical activity.

The mean follow-up was $12.8 \pm 5.2$ months (range 6-18). Data were collected at the outpatient clinic 5 days after surgery, 15 days after surgery and then 1,3 , 6,12 months postoperatively for the first year, twice in the second year. In 2015, all patients were visited at the outpatient clinic or were telephoned if not available to visit the clinic. Data was analyzed in January 2016. Statistical analyses was performed using IBM SPSS version 20 for Windows. Continuous variables are described as mean and standard deviation (SD), whereas categorical variables were described as number and percentage.

Baseline demographic factors are summarized in table 1.

\section{RESULTS}

All 111 patients who underwent bariatric surgery were suitable for early discharge, between $24-72$ hours. Mean operative time ( $\mathrm{min}$ ) for $\mathrm{LSG}$ ( $\mathrm{n}=48$ patients) was $84.72 \pm 14.4$ and for LAGB ( $n=63$ patients) $75.88 \pm 12.8$. Length of hospital stay (LOS), expressed as mean standard deviation \pm SD (days) was $2.04 \pm 0.96$ days for LSG and $0.89 \pm 0.61$ for LAGB. Regarding short term complications (within 30 days post-operatively) for the 48 patients who underwent LSG, $6(12,5 \%)$ had raised temperature, $1(2 \%)$ bled, $10(20 \%)$ patients developed PONV, $1(2 \%)$ had ileus, $2(4,1 \%)$ got gastro-esophageal reflux, $1(2 \%)$ got abdominal wall haematoma, $2(4.1 \%)$ had diarrhea. Mortality rate was $0 \%$, reoperation rate was $0 \%$ and hospital readmission rate $2 \%$ pertaining to one patient who was readmitted for sickness. Concerning short term complications in 63 patients who underwent LAGB the commonest, representing $3 \%$, was two patients developed arise of temperature, no bleeding or paralytic ileum or reflux was recorded. One patient (1.5\%) developed PONV and one (1.5\%) patient diarrhea. Hospital readmission rate, reoperation rate and mortality are $0 \%$. (table 2 and table 3 ) 
Table 1 - Patient's baseline characteristics

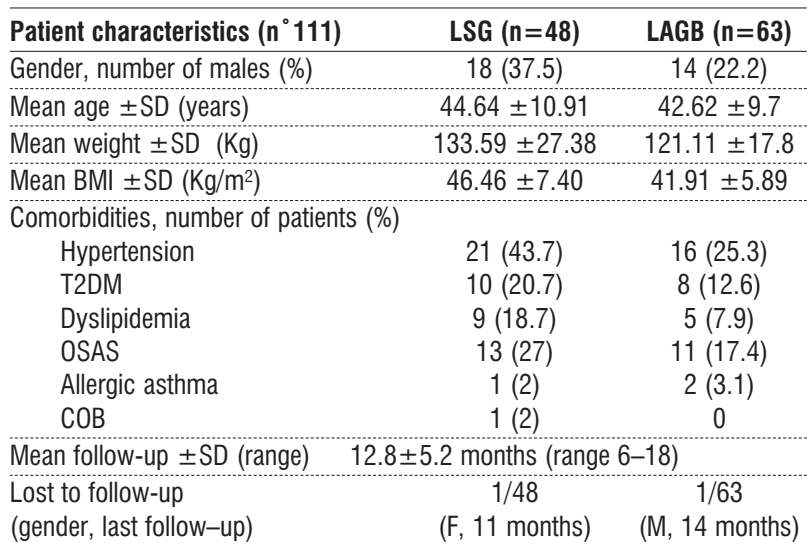

Abbreviations: LSG laparoscopic sleeve gastrectomy, LAGB laparoscopic adjustable gastric banding, M male, F female, SD standard deviation, T2DM type 2 diabetes mellitus, OSAS obstruction sleep apnea syndrome, COB chronic obstructive broncho-pneumopathy

\begin{tabular}{lcc}
\hline & LSG $(\mathbf{n}=\mathbf{4 8})$ & LAGB $(\mathbf{n}=\mathbf{6 3})$ \\
\hline Mean operative time (min) & $84.72 \pm 14.4$ & $75.88 \pm 12.8$ \\
\hline $\begin{array}{l}\text { Length of hospital stay, } \\
\text { mean } \pm \text { SD (days) }\end{array}$ & $2,04 \pm 0.96$ & $0.89 \pm 0.61$ \\
\hline
\end{tabular}

\section{DISCUSSION}

The majority of ERAS data has been derived from patients undergoing colorectal surgery (5), with less clear data on the application of ERAS in bariatric surgery $(8,9)$. An observational case series including 4400 patients undergoing LRYGB over a 4-year period reported a decrease in the mean hospital LOS from 2 days to 1 day, with a $3.4 \%$ postoperative complication rate, $60 \%$ of which were within the first 24 hours (10). A further study of 200 patients undergoing LRYGB, LSG, and laparoscopic adjustable gastric band (LAGB) and enrolled in ERAS principles found successful discharge on the first post-operative day in 37\% of LRYGB and $28 \%$ of LSG patients (11). In this early stage of implementation of our protocol other factors may have influenced the rate of discharge on the first postoperative day, including patient willingness to go home, poor pain control, postoperative nausea and vomiting, difficulty during the procedure, and the need for highdependency care (e.g., patients with obstructive sleep apnea). To facilitate early discharge of patients after bariatric surgery, patient performance status needs optimization and expectations need to be adequately managed preoperatively in a multidisciplinary setting with education (15). Oral fluid intake is started in the immediate postoperative period, and patient mobilization is also commenced a few hours after the end of the operation

Inherent difficulties in the clinical assessment of the
Table 2

\begin{tabular}{lcc}
\hline $\begin{array}{l}\text { Short-term complications } \\
\text { (within } \mathbf{3 0} \text { days } \\
\text { postoperatively) }\end{array}$ & $\begin{array}{c}\text { Sleeve } \\
\text { (n. pz }=\mathbf{2 0} / \mathbf{4 8}) \\
\text { frequency (\%) }\end{array}$ & $\begin{array}{c}\text { LAGB } \\
\text { (n. pz= 3/63) } \\
\text { frequency (\%) }\end{array}$ \\
\hline Fevor & $6(12.5)$ & $2(3.1)$ \\
\hline $\begin{array}{l}\text { Bleeding requiring transfusion } \\
\text { of at least one unit of blood }\end{array}$ & $1(2)$ & 0 \\
\hline vomiting & $10(20)$ & $1(1.5)$ \\
\hline Gastric Fistula & 0 & 0 \\
\hline Delay in Drainage & $1(2)$ & 0 \\
\hline Gastro-intestinal Reflux & $2(4.1)$ & 0 \\
\hline Abdominal wall Haematoma & $1(2)$ & $1(1.5)$ \\
\hline Diarrhoea & $2(4.1)$ & 0 \\
\hline Re-operation rate & 0 & 0 \\
\hline Hospital re-admissions rate & $1(2)$ for vomiting & 0 \\
\hline Death rate & 0 & 0 \\
\hline
\end{tabular}

Table 3

\begin{tabular}{lcc}
\hline Long-term complications & $\begin{array}{c}\text { Sleeve } \\
\text { (n. pz }=\mathbf{1 1 / 4 8 )} \\
\text { frequency (\%) }\end{array}$ & $\begin{array}{c}\text { LAGB } \\
\text { (n. pz=5/63) } \\
\text { frequency (\%) }\end{array}$ \\
\hline Dysphagia & $1(2)$ & $1(1.5)$ \\
\hline Gastric Ulceration & 0 & $1(1.5)$ \\
\hline Lap Band Erosion & $/$ & $1(1.5)$ \\
\hline Lap Band Slippage & $3(6.2)$ & 0 \\
\hline Anaemia & $2(4.1)$ & 0 \\
\hline Gastro-intestinal Reflux & $1(2)$ & $1(1.5)$ \\
\hline Cholelithiasis gallstones & $3(6.2)$ & 0 \\
\hline Vomiting & $1(2)$ & 0 \\
\hline Constipation & $1(2)$ & 0 \\
\hline Hair loss & $1(2)$ & $1(1.5)$ \\
\hline Oesophageal Stenosis & $1(2)$ & 0 \\
\hline Cerebral Ischaemia & 0 & 0 \\
\hline Re-operation rate & 0 & 0 \\
\hline Hospital re-admissions rate & 0 & 0 \\
\hline Death rate & & 0 \\
\hline
\end{tabular}

"acute abdomen" in obese patients mean that a higher level of suspicion is necessary for the identification of patients who are not following the usual pathway of recovery and any deviation from the norm indicates that the patient should be kept in hospital for further assessment and/or imaging, with a low threshold for computed tomographic (CT) scanning or relaparoscopy. The ERAS program in our institution was designed and introduced by one senior surgeon and the head of anesthesia and intensive care department, with the help of psychiatrists and dieticians. The protocol was developed after a literature review of ERAS in colorectal surgery.

In our experience, preoperative patient preparation, psychiatric structured anaesthetic protocols, and postoperative care with recurrent visit to patient's bedside, an adequate pain control and monitoring of vital parameters play an essential role in the achievement of 
patient discharge within 24-72 hours.

We acknowledge that postoperative LOS is only a surrogate marker of recovery and does not necessarily reflect the true recovery of a patient but ERAS definitely improves the overall patient experience.

\section{CONCLUSION}

This study has demonstrated that applying an ERABS protocol is feasible, safe, associated with low morbidity, acceptable LOS and low 30-day hospital readmission rates. The presence of multiple medical comorbidities should not preclude use of such a protocol within bariatric patients. Further prospective studies are required to reduce further more LOS and costs associated.

\section{REFERENCES}

1. Wilmore DW, Kehlet $H$. Management of patients in fast track surgery. BMJ. 2001 Feb 24;322(7284):473-6.

2. Kehlet $\mathrm{H}$, Wilmore DW. Multimodal strategies to improve surgical outcome. Am J Surg. 2002 Jun;183(6):630-41.

3. Kehlet H, Dahl JB. Anaesthesia, surgery, and challenges in postoperative recovery. Lancet. 2003 Dec 6;362(9399):1921-8.

4. Lassen K, Soop M, Nygren J, Cox PB, Hendry PO, Spies C, et al. Consensus review of optimal perioperative care in colorectal surgery: Enhanced Recovery after surgery (ERAS) Group recommendations. Arch Surg. 2009 0ct;144(10):961-9. doi: 10.1001/ archsurg.2009.170.

5. Wind J, Polle SW, Fung Kon Jin PH, Dejong CH, von Meyenfeldt MF,
Ubbink DT, et al. Systematic review of enhanced recovery programmes in colonic surgery. Br J Surg. 2006 Jul;93(7):800-9.

6. Podore PC, Throop EB. Infrarenal aortic surgery with a 3-day hospital stay: A report on success with a clinical pathway. J Vasc Surg. 1999 May;29(5):787-92.

7. Tovar EA, Roethe RA, Weissig MD, Lloyd RE, Patel GR. One-day admission for lung lobectomy: an incidental result of a clinical pathway. Ann Thorac Surg. 1998 Mar;65(3):803-6.

8. Bamgbade OA, Adeogun BO, Abbas K. Fast-track laparoscopic gastric bypass surgery: outcomes and lessons from a bariatric surgery service in the United Kingdom. Obes Surg. 2012 Mar;22(3):398-402. doi: 10.1007/s11695-011-0473-3.

9. Lemanu DP, Singh PP, Berridge K, Burr M, Birch C, Babor R, et al. Randomized clinical trial of enhanced recovery versus standard care after laparoscopic sleeve gastrectomy. Br J Surg. 2013 Mar;100(4): 482-9. doi: 10.1002/bjs.9026. Epub 2013 Jan 21.

10. Varadhan KK, Neal KR, Dejong CH, Fearon KC, Ljungqvist O, Lobo DN. The enhanced recovery after surgery (ERAS) pathway for patients undergoing major elective open colorectal surgery: a meta-analysis of randomized controlled trials. Clin Nutr. 2010 Aug;29(4):434-40. doi: 10.1016/j.cInu.2010.01.004. Epub 2010 Jan 29.

11. Lemanu DP, Singh PP, Berridge K, Burr M, Birch C, Babor R, et al. Randomized clinical trial of enhanced recovery versus standard care after laparoscopic sleeve gastrectomy. Br J Surg. 2013 Mar;100(4): 482-9. doi: 10.1002/bjs.9026. Epub 2013 Jan 21.

12. Kiecolt-Glaser JK, Page GG, Marucha PT, MacCallum RC, Glaser $R$. Psychological influences on surgical recovery: perspectives from psychoneuroimmunology. Am Psychol. 1998 Nov;53(11): 1209-18.

13. Egbert LD, Battit G, Welch C, Bartlett M. Reduction of postoperative pain by encouragement and instruction of patients: a study of doctorpatient rapport. N Engl J Med. 1964 Apr 16;270:825-7.

14. Halaszynski TM, Juda R, Silverman DG. Optimizing postoperative outcomes with efficient preoperative assessment and management. Crit Care Med. 2004 Apr;32(4 Suppl):S76-86.

15. Blay N, Donoghue J. The effect of pre-admission education on domiciliary recovery following laparoscopic cholecystectomy. Aust J Adv Nurs. 2005 Jun-Aug;22(4):14-9. 